\title{
Effects of Ropivacaine Concentration on Analgesia After Ultrasound-Guided Serratus Anterior Plane Block: A Randomized Double-Blind Trial
}

This article was published in the following Dove Press journal: Journal of Pain Research

\section{Lvdan Huang* \\ Liangyu Zheng* \\ Bingjing $\mathrm{Wu}^{*}$ \\ Zhengjie Chen \\ Jiali Chen \\ Xuzhong Xu \\ Kejian Shi}

Department of Anesthesiology, The First Affiliated Hospital of Wenzhou Medical University, Wenzhou, Zhejiang, People's Republic of China

*These authors contributed equally to this work
Correspondence: Kejian Shi; Xuzhong Xu Department of Anesthesiology, The First Affiliated Hospital of Wenzhou Medical University, South Baixiang Town,

Wenzhou, Zhejiang, People's Republic of

China

Tel +86 I3868889697;

$+8613706657799$

Fax +86 55578999 Ext 669697;

+8655578999 Ext 667799

Email wzshikejian@163.com;

xuzhong@263.net
Purpose: Serratus anterior plane (SAP) block is effective for analgesia after breast surgery. Whether a higher local anesthetic concentration prolongs sensory block duration and improves postoperative analgesia remains unclear. The aim of this study was to compare the analgesic effects of SAP block with different concentrations of ropivacaine.

Patients and Methods: Sixty patients scheduled for breast surgery were enrolled in this randomized double-blind trial. SAP block was induced with $20 \mathrm{~mL}$ of $0.375 \%, 0.5 \%$, or $0.75 \%$ ropivacaine in Group R0.375, Group R0.5, and Group R0.75, respectively. The primary endpoint was the area under the curve (AUC) of numerical rating scale (NRS) pain intensity scores at rest over time. The secondary endpoints were AUC of NRS pain intensity scores on movement over time, active sensory block duration, tramadol consumption, and the elapsed time between completion of surgery and the first administration of rescue analgesia.

Results: The AUC of NRS pain intensity scores at rest of Group R0.375 was significantly higher than that of Groups R0.5 and R0.75 ( $P=0.025$, and $P=0.001)$. The AUC of NRS pain intensity scores on movement of Group R0.375 was also significantly higher than that of Groups R0.5 and R0.75 (both $P<0.001)$. At higher ropivacaine concentrations, the duration of SAP sensory block increased $(P<0.001)$. Tramadol consumption and the elapsed time between completion of surgery and the first administration of rescue analgesia were similar in the three groups $(P>0.05)$.

Conclusion: A comparison of $0.5 \%$ and $0.75 \%$ ropivacaine showed no significant difference in postoperative analgesia, but both were superior to $0.375 \%$ ropivacaine, although higher ropivacaine concentration lengthened the duration of SAP block. Therefore, SAP block with $0.5 \%$ ropivacaine is recommended for postoperative analgesia in breast surgery. Keywords: analgesia, ultrasound, regional anesthesia, breast

\section{Introduction}

Local anesthetics (LAs) injection into the fascial plane deep beneath the serratus anterior muscle in the lateral chest wall can block cutaneous branches of the intercostal nerves ${ }^{1}$ and provide effective postoperative analgesia for procedures such as breast surgery, ${ }^{2}$ video-assisted thoracoscopic surgery, ${ }^{3,4}$ transthoracic esophagectomy, ${ }^{5}$ and infant thoracotomy. ${ }^{6}$ Kunigo et al reported that serratus anterior plane (SAP) block with $40 \mathrm{~mL}$ of $0.375 \%$ ropivacaine diffuses to a greater extent than $20 \mathrm{~mL}$ ropivacaine in the craniocaudal direction, but the time to first need of analgesic rescue was not extended by a larger injection volume. ${ }^{7}$ Sensory block in the thoracic region by SAP block with $20 \mathrm{~mL}$ LAs can meet the analgesia needs for breast surgery. ${ }^{8}$ Increasing LAs concentration may 
be a better approach to improve the analgesic efficacy of SAP block and prolong the duration of pain relief.

No studies have compared the efficacies of different concentrations of LAs in SAP block. However, similar investigations for other types of regional anesthesia showed that analgesia duration was associated with an adequate quantity of LAs during lumbar plexus block or brachial plexus block. ${ }^{9,10}$ Wong et al reported that the LAs concentration in brachial plexus block was inversely proportional to the tidal volume, with a high concentration leading to some respiratory complications. ${ }^{11}$ Therefore, appropriate LAs concentration during regional anesthesia is important to provide sufficient analgesia and avoid adverse reactions caused by excessive concentration.

Ropivacaine is used widely in regional anesthesia as one of long-acting local anesthetics, because of its relatively larger maximum dosage and relatively lower systemic toxicity and neurotoxicity, compared with bupivacaine. The present study was designed to compare different concentrations of ropivacaine on SAP block for postoperative analgesia in patients undergoing breast surgery. The aim was to compare the effects of SAP block for three ropivacaine concentrations. The primary endpoint was the area under the curve (AUC) of the numerical rating scale (NRS) pain intensity scores at rest over time. The secondary endpoints were the AUC of NRS pain intensity scores on movement over time, NRS scores at individual time points, worst pain score, incidence of moderate-severe pain (NRS $>3$ ), active sensory block duration, postoperative analgesic consumption, the elapsed time between surgery completion and the first administration of rescue analgesia, and the incidence of adverse events.

\section{Materials and Methods}

This study was approved by the Ethics Committee of The First Affiliated Hospital of Wenzhou Medical University (No. 12, 2018) and written informed consent was obtained from all participants. The trial was registered prior to patient enrollment at the Chinese Clinical Trial Registry (ChiCTR1800015927). A CONSORT checklist was used for patient enrollment and allocation (Figure 1). Sixty female patients scheduled to undergo breast surgery for fibroadenoma or intraductal papilloma were enrolled in the study. Inclusion criteria included age between 18 and 65 years, American Society of Anesthesiologists (ASA) physical status score of I or II, and body mass index (BMI) between 18 and $28 \mathrm{~kg} / \mathrm{m}^{2}$. Exclusion criteria included an allergy to ropivacaine or other drugs used in this trial, a history of alcohol or analgesic dependence, peripheral neuropathy (eg diabetic neuropathy), coagulopathy, skin pathology at the puncture site, difficulty with communication, or unwillingness to receive regional block anesthesia.

Intravenous access was established and routine monitors (noninvasive blood pressure, continuous electrocardiogram, and pulse oximetry) were applied in the operating room. Patients were randomized into three groups: R0.375, R0.5, and $\mathrm{R} 0.75$ ( $\mathrm{n}=20$ per group) according to a random number table that was generated by SPSS 22.0 statistical software (IBM Corporation, Armonk, NY, USA). Experimental drugs were prepared by a researcher who was not involved in follow-up assessments. Group R0.375 received $20 \mathrm{~mL}$ of $0.375 \%$ ropivacaine (LBKL; AstraZeneca AB, Sweden), Group R0.5 received $20 \mathrm{~mL}$ of $0.5 \%$ ropivacaine, and Group $\mathrm{R} 0.75$ received $20 \mathrm{~mL}$ of $0.75 \%$ ropivacaine.

An ultrasound-guided deep SAP block was performed by an attending anesthesiologist, who was blinded to patient grouping and ropivacaine concentration and did not participate in data collection or analysis. Patients were placed in the lateral decubitus position with the operation side up. Midazolam $1 \mathrm{mg}$ and fentanyl $50 \mu \mathrm{g}$ were intravenously administered for sedation and analgesia. After routine disinfection and towel draping, a high-frequency (6 to $12 \mathrm{MHz}$ ) linear probe (SonoSite X-Porte; SonoSite Inc., Bothell, WA, USA) was placed over the middle clavicular region in a sagittal plane. The ribs were counted to identify the fifth rib in the midaxillary line. The subcutaneous tissue, latissimus dorsi, serratus anterior, intercostal muscle, and pleura were identified at 4-5 intercostal in the midaxillary line. Using an in-plane technique, a $22 \mathrm{G}$ needle 1 to $2 \mathrm{~cm}$ medial to the probe was inserted from the cranial to caudal direction into the plane deep underneath the serratus muscle. The needle tip location was confirmed by injecting 1 to $2 \mathrm{~mL}$ saline, then LAs were injected into the fascial plane (Figure 2).

We defined a successful active sensory block as a lost or markedly reduced perception of cold. ${ }^{12}$ Thirty minutes after ropivacaine injection, anesthesiologist who did the SAP block confirmed the loss of the dermatomal sensory. All patients underwent general anesthesia performed by attending anesthesiologists who were not involved in the study and blinded to grouping. The same group surgeons completed all the surgeries. Propofol (1 to $2 \mathrm{mg} / \mathrm{kg}$ ), sufentanil $(0.3 \mu \mathrm{g} / \mathrm{kg}$ ), and rocuronium $(0.6 \mathrm{mg} / \mathrm{kg})$ were used for induction and the laryngeal mask was used to control the airway during surgery. Anesthesia was maintained using propofol (2 to $\left.6 \mathrm{mg} \cdot \mathrm{kg}^{-1} \cdot \mathrm{h}^{-1}\right)$, remifentanil $\left(0.1\right.$ to $\left.0.2 \mu \mathrm{g} \cdot \mathrm{kg}^{-1} \cdot \mathrm{min}^{-1}\right)$, and sevoflurane ( $1 \%$ to $2 \%$ in oxygen), and the bispectral index value was maintained at 40 to 60 . Tropisetron $(5 \mathrm{mg})$ and 


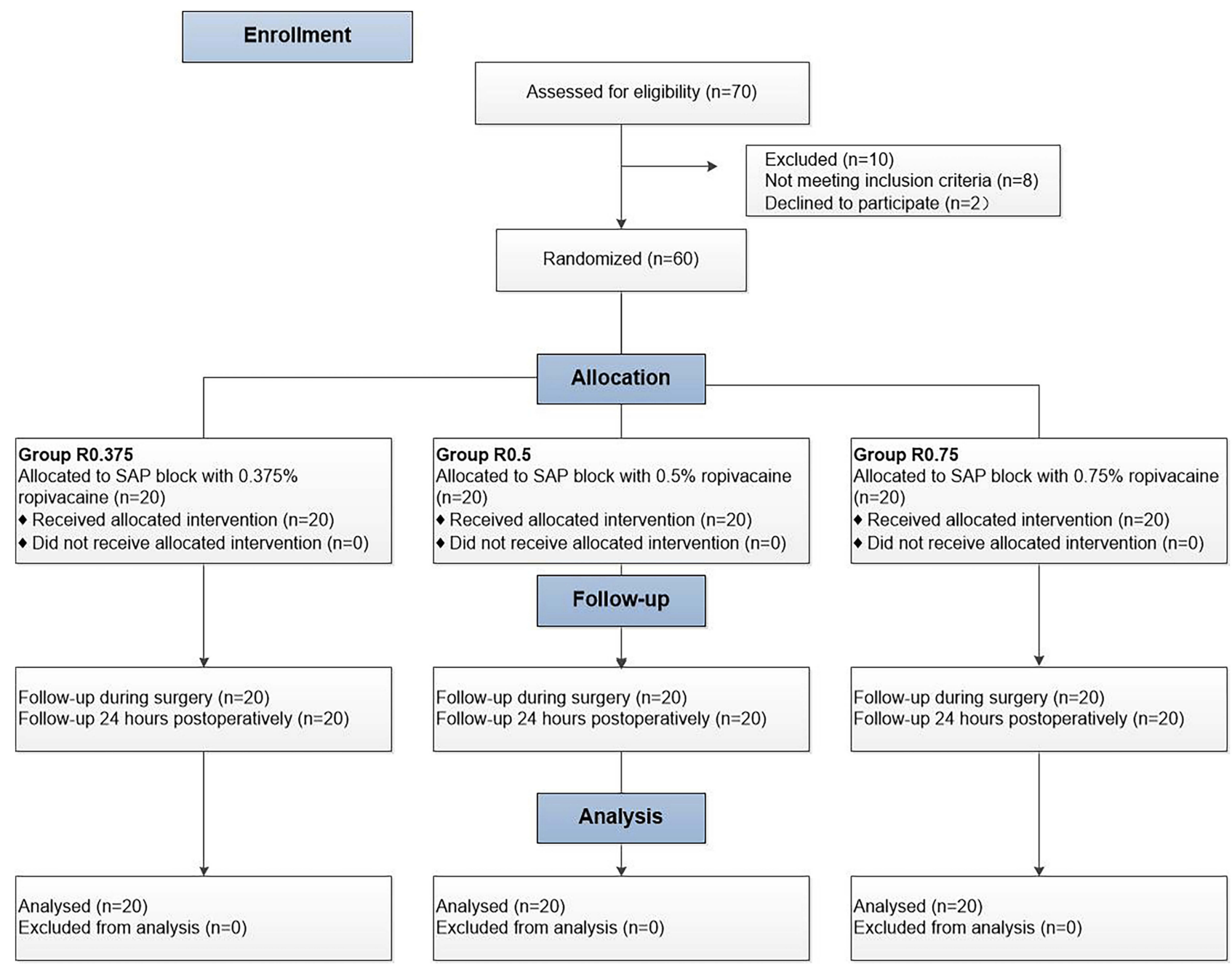

Figure I CONSORT flow diagram.

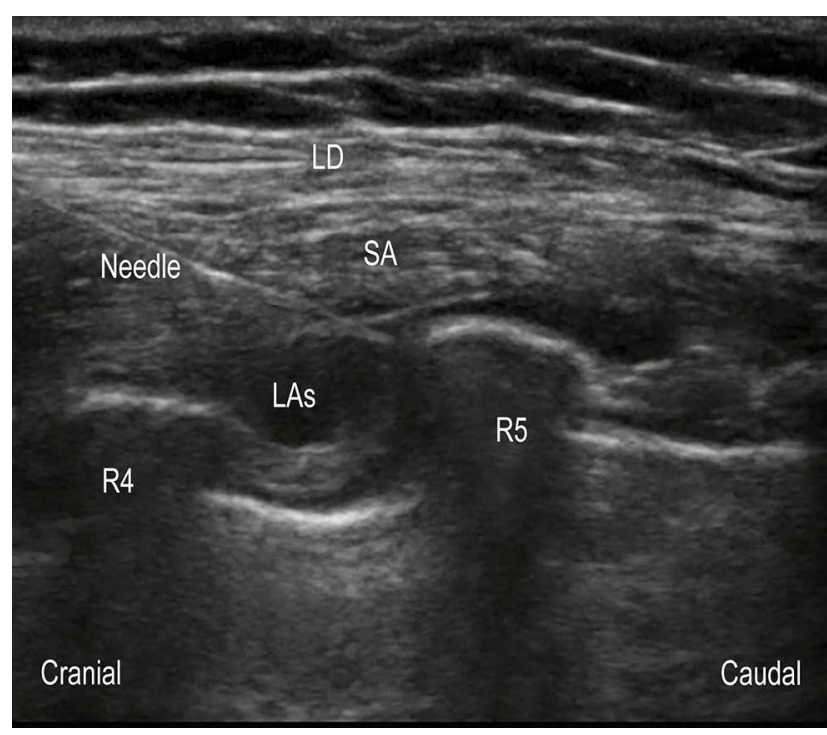

Figure 2 Ultrasonogram of serratus anterior plane block.

Abbreviations: LD, latissimus dorsi muscle; SA, serratus anterior; LAs, local anesthetics; R4, the fourth rib; R5, the fifth rib. flurbiprofen $(100 \mathrm{mg})$ were intravenously administered about 30 mins before the end of the surgery. Tramadol $50 \mathrm{mg}$ was given intravenously for analgesic relief after the surgery when analgesia was insufficient (NRS $\geq 4$ ).

\section{Data Collection}

Follow-up was performed at 1, 2, 6, 12, and 24 hrs postoperatively. At each evaluation, patients were asked to report their NRS pain scores ranging from 0 to 10 both at rest and on movement $(0=$ no pain, $10=$ worst imaginable pain $)$. Pain on movement was defined as the pain feeling when sitting forward from a recumbent position. The worst pain scores within $24 \mathrm{hrs}$, incidence of moderate-severe pain (NRS $>3$ ), time to first request of rescue analgesics, and total consumption of rescue analgesics were recorded. After surgery, sensory block was measured every $2 \mathrm{hrs}$ by cold stimulus until it was completely resolved. Nausea and vomiting, itching, chills, 
hematoma, infection, and hypoesthesia and other adverse events were documented. All postoperative evaluations and record-keeping were performed by an independent investigator, who was not involved in any other process of the trial.

\section{Statistical Analysis}

All data were analyzed using SPSS 22.0 statistical software. They were tested for normal distribution using the ShapiroWilk test. For each patient, their AUC of NRS pain intensity scores both on movement and at rest was calculated by multiplying the time interval with the NRS score using GraphPad Prism version 7 (GraphPad Software Inc., San Diego, CA, USA). Measured data with normal distributions are expressed as mean(standard deviation), non-normally distributed data are expressed as median (interquartile range), and categorical data are expressed as n (\%). Mean age, height, weight, BMI, surgery duration, AUC of NRS pain intensity scores both on movement and at rest, and SAP block duration were analyzed using oneway analyses of variance. The post hoc Bonferroni test was used for multiple two-group comparisons. The incidence of moderate-severe pain (NRS $>3$ ) and adverse events were determined using chi-square tests or Fisher's exact tests as appropriate. The elapsed time between surgery completion and the first administration of rescue analgesia were plotted as KaplanMeier survival curves and compared using the Log rank tests. ASA classification, NRS scores at individual time points, the worst pain scores within $24 \mathrm{~h}$ and total consumption of rescue analgesics were compared with Kruskal-Wallis H-tests. Multiple testing was performed using Mann-Whitney $U$ tests. $P<0.05$ was deemed statistically significant. To reduce type I error, $P<0.017$ was considered statistically significant after Bonferroni correction when comparing two groups.

\section{Sample Size Calculation}

The primary endpoint was the AUC of NRS pain intensity scores at rest over time. In the preliminary study, 15 patients were assigned to Groups R0.375, R0.5, and $\mathrm{R} 0.75(\mathrm{n}=5)$, and the AUC of NRS pain intensity scores at rest were $70.4 \pm 7.6,60.9 \pm 8.9$, and $59.5 \pm 9.0$, respectively. A sample size of 16 per group was obtained by PASS 11.0 (NCSS Statistical Software, Kaysville, UT, USA) with $\alpha=0.05$ and $\beta=0.1$. We planned to recruit 20 patients per group considering the loss potential and errors.

\section{Results}

There were no statistically significant differences between groups in ASA classification, mean age, height, weight, BMI, or surgery duration $(P>0.05)$ (Table 1$)$.
Table I Demographic and Intraoperative Characteristics

\begin{tabular}{|l|l|l|l|l|}
\hline \multirow{2}{*}{ Characteristic } & $\mathbf{R 0 . 3 7 5}$ & $\mathbf{R 0 . 5}$ & $\mathbf{R 0 . 7 5}$ & \multirow{2}{*}{-value } \\
\cline { 2 - 4 } & $\mathbf{( n = 2 0 )}$ & $\mathbf{( n = 2 0 )}$ & $\mathbf{( n = 2 0 )}$ & \\
\hline ASA classification(I/II) & $13 / 7$ & $15 / 5$ & $16 / 4$ & 0.556 \\
Age, y & $43.5(8.5)$ & $41.0(14.8)$ & $39.0(9.6)$ & 0.449 \\
Height, cm & $158.7(4.3)$ & $158.2(4.0)$ & $159.0(4.2)$ & 0.809 \\
Weight, kg & $58.8(8.0)$ & $60.0(7.5)$ & $58.2(6.3)$ & 0.859 \\
BMI, kg/m ${ }^{2}$ & $23.3(2.8)$ & $23.8(2.7)$ & $23.1(2.9)$ & 0.746 \\
Surgical duration, min & $58.3(12.0)$ & $61.5(15.6)$ & $57.8(13.4)$ & 0.646 \\
SAP block duration, h & $7.8(2.3)$ & $11.0(3.3)$ & $13.9(4.0)$ & $<0.001$ \\
\hline
\end{tabular}

Note: Measured data with normal distribution were expressed as mean (standard deviation). Abbreviations: ASA, American Society of Anesthesiologists; BMI, body mass index; SAP, serratus anterior plane.

\section{Pain Scores}

The AUCs of NRS pain intensity scores both at rest and on movement through $24 \mathrm{~h}$ postsurgery are shown in Figure 3A and B. The AUCs of NRS pain intensity scores at rest were 64.1 $\pm 17.4,50.0 \pm 15.0$, and 44.3 \pm 16.2 in Groups R0.375, R0.5, and R0.75, respectively. The AUC of NRS pain intensity scores at rest of Group R 0.375 was significantly higher than that of Groups R 0.5 and $\mathrm{R} 0.75$ ( $P=0.025$ and $P=0.001$, respectively). The AUCs of NRS pain intensity scores on movement were $71.8 \pm 12.3,53.0 \pm 15.9$, and $48.2 \pm 13.3$ in Groups R0.375, R 0.5 , and R 0.75 , respectively. The AUC of NRS pain intensity scores on movement of Group R0.375 was significantly higher than those of Groups R0.5 and R0.75 (both $P<0.001$ ). There was no significant difference in AUC of NRS pain intensity scores between Groups R 0.5 and R0.75, either at rest or on movement ( $P=0.809$ and $P=0.849$, respectively).

The NRS scores at rest and on movement over $24 \mathrm{hrs}$ were shown in Figure $3 \mathrm{C}$ and D. There was no significant difference in NRS scores at rest between the three groups at 1, 2, and 6 h postoperatively $(P=0.387, P=0.101$, and $P=0.066$, respectively). Group R 0.375 had significantly higher NRS scores at rest at $24 \mathrm{~h}$ when compared with Group R0.5 $(P=0.002)$, and at 12 and $24 \mathrm{~h}$ when compared with Group R0.75 ( $P=0.010$ and $P<0.001)$. There were no significant differences in NRS scores at rest between Groups R0.5 and R0.75 at any time point ( $P=0.289, P=0.060, P=0.602, P=0.461$, and $P=0.289$, respectively). There were also no significant differences in NRS scores on movement between the three groups at 1 and 2 h postoperatively $(P=0.367$ and $P=0.055)$. Group R0.375 had significantly higher NRS scores on movement at 6,12 , and $24 \mathrm{~h}$ compared with Group R0.5 ( $P=0.009, P=0.001$, and $P<0.001$, respectively) and Group R0.75 ( $P=0.009, P<0.001$, and $P<0.001$, respectively). There were no significant differences in NRS scores on movement between Groups R0.5 and 

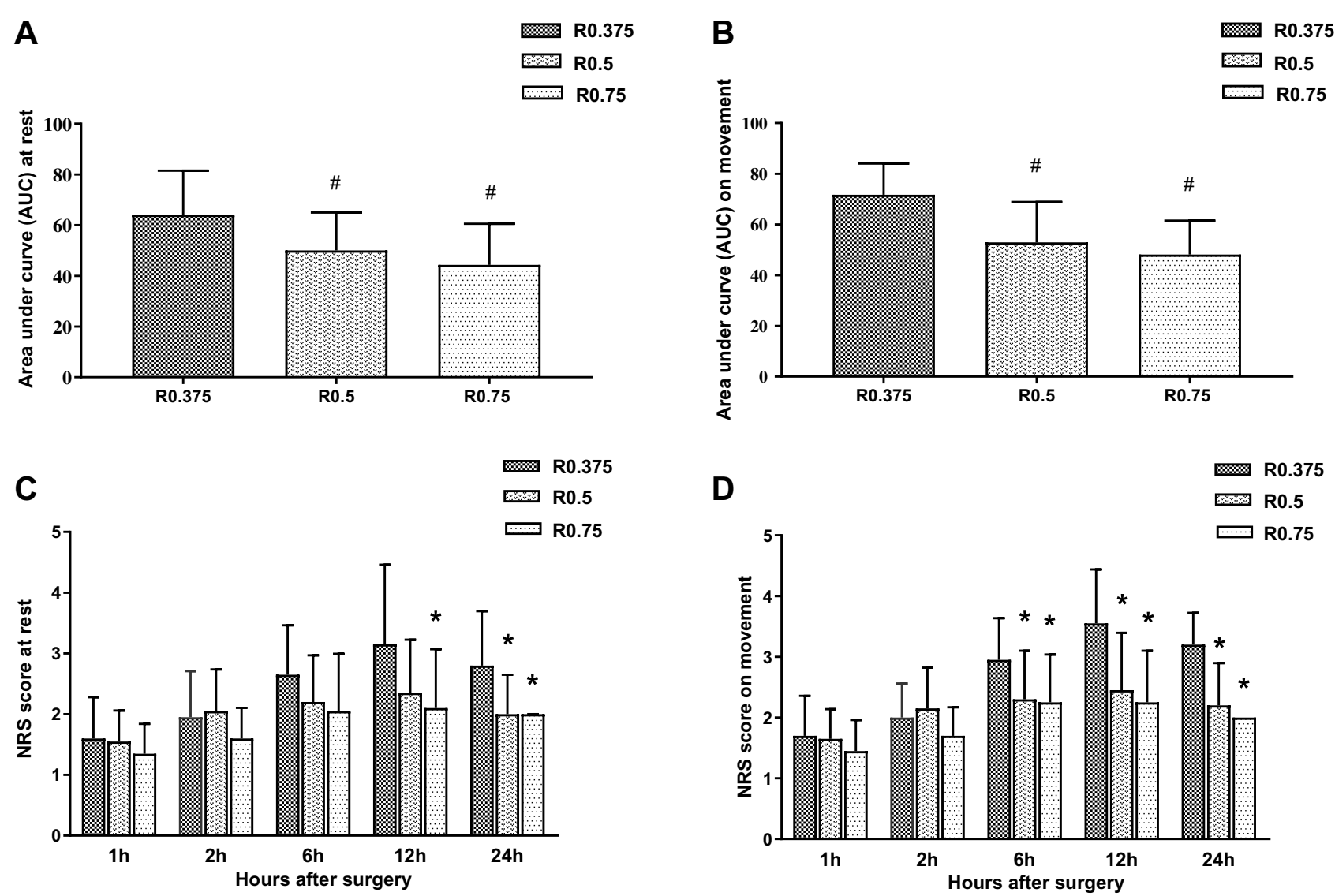

Figure 3 (A) AUC of NRS pain intensity scores at rest through $24 \mathrm{~h}$ postsurgery; (B) AUC of NRS pain intensity scores on movement through $24 \mathrm{~h}$ postsurgery. (C) NRS score (ranging from 0 to 10) for pain at rest; (D) NRS score (ranging from 0 to 10) for pain on movement. Values are mean (standard deviation) or median (interquartile range), $\mathrm{n}=20$. R0.375: $0.375 \%$ ropivacaine with $20 \mathrm{~mL}$ volume; R0.5: $0.5 \%$ ropivacaine with $20 \mathrm{~mL}$ volume; $\mathrm{R} 0.75$ : $0.75 \%$ ropivacaine with $20 \mathrm{~mL}$ volume. ${ }^{\#} P<0.05$ indicates a significant difference in comparison to Group R0.375. $* P<0.017$ indicates a significant difference in comparison to Group R0.375.

Abbreviation: NRS, numerical rating scale.

R0.75 at any time point $(P=0.289, P=0.052, P=0.968$, $P=0.565$, and $P=0.277$, respectively).

The worst pain scores and incidence rates of moderatesevere pain (NRS $>3$ ) of the three groups are shown in Figure 4. The worst pain scores (both at rest and on movement) in Group R0.375 were significantly higher compared to Group R0.5 $(P<0.001$ and $P=0.003)$ and Group R0.75 (both $P<0.001$ ), while NRS scores (both at rest and on movement) of Groups R 0.5 and R0.75 were not significantly different ( $P=0.630$ and $P=0.360$ ). The incidence of moderate-severe pain at rest in Group R0.375 was significantly higher than that in Groups R0.5 and R0.75 (both $P=0.013$ ), but there was no significant difference between Groups R0.5 and R0.75 $(P=1.000)$. The incidence of moderate-severe pain on movement in Group R0.375 was significantly higher than in Group R0.75 ( $P=0.006$ ), but there was no significant difference compared to Group R0.5 $(P=0.018)$. There was no significant difference in the incidence of moderate-severe pain on movement between Groups R0.5 and R0.75 $(P=1.000)$.

\section{Duration of SAP Block}

The duration of SAP block was significantly different among the three groups $(P<0.001)$. The block duration of Group R0.375 was significantly shorter than that of Groups R0.5 and R0.75 ( $P=0.010$ and $P<0.001$, respectively), and Group R0.75 had a prolonged block duration compared to Group R0.5 ( $P=0.022)$ (Table 1).

\section{Postoperative Analgesics and the Time of First Need for Tramadol}

The median (interquartile range) of tramadol consumption levels in the first $24 \mathrm{~h}$ after surgery were $50(0-50)$ $\mathrm{mg}, 25(0-50) \mathrm{mg}$, and $0(0-50) \mathrm{mg}$ in Groups R0.375, $\mathrm{R} 0.5$, and R0.75, respectively. Postoperative tramadol dosages were not significantly different $(P=0.478)$. The elapsed times between completion of surgery and the first administration of rescue analgesia are shown in Figure 5. There was no significant difference among the three groups $(P=0.180)$. 

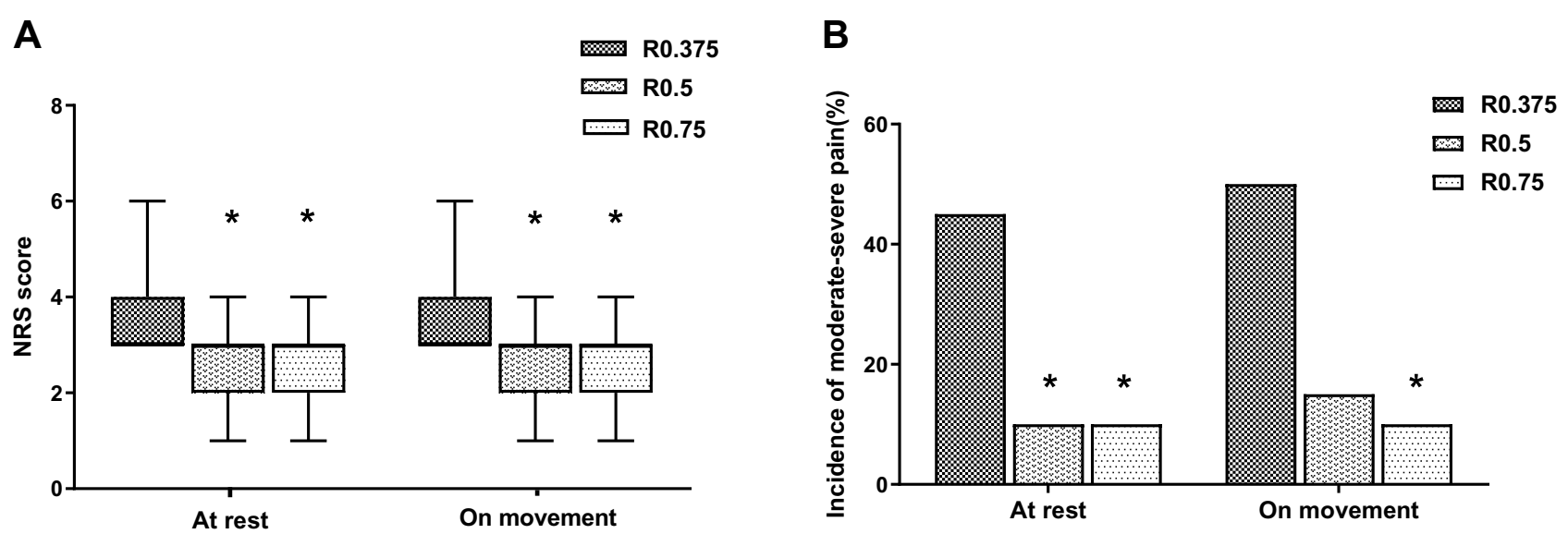

Figure 4 (A) the worst pain scores (ranging from 0 to 10); (B) the incidence of moderate-severe pain (NRS $>3$ ). Values are median (interquartile range) or percentage, $n=20$. R0.375: $0.375 \%$ ropivacaine with $20 \mathrm{~mL}$ volume; $\mathrm{R} 0.5$ : $0.5 \%$ ropivacaine with $20 \mathrm{~mL}$ volume; $\mathrm{R} 0.75$ : $0.75 \%$ ropivacaine with $20 \mathrm{~mL}$ volume. $* P<0.017$ indicates a significant difference in comparison to Group R0.375.

Abbreviation: NRS, numerical rating scale.

\section{Adverse Events}

The incidence rates of nausea and vomiting were $6(30 \%)$, 5 (25\%), and 5 (25\%), in Groups R0.375, R0.5, and R0.75, respectively $(P=0.918)$. No other adverse effects were observed in the three groups.

\section{Discussion}

This study compared the duration and postoperative analgesic effect of SAP block with different concentrations of ropivacaine for breast surgery. The results showed that the analgesic effects of $0.75 \%$ and $0.5 \%$ ropivacaine were better than that of $0.375 \%$ ropivacaine for breast surgery in the first $24 \mathrm{~h}$ after surgery, but there was no significant difference between $0.75 \%$ and $0.5 \%$ ropivacaine. The duration of sensory block was prolonged with higher concentrations. Postoperative analgesic consumption and the elapsed time between surgery completion and the first administration of rescue analgesia were similar among the three groups.

LAs injection into the SAP blocks the lateral cutaneous branch of the thoracic intercostal nerve, providing effective postoperative analgesia for pain associated with an incision in the anterolateral thoracic wall. ${ }^{8,13,14}$ The breast skin and subcutaneous tissue are generally considered to be innervated by the intercostal nerve from the 2 nd to the 6th rib. Other reports stated that the dermatomal paresthesia of an SAP block at the 5th costal level could reach T2T9, meeting the analgesic requirements for breast surgery. ${ }^{8,15}$ In the present investigation, $20 \mathrm{~mL}$ of ropivacaine was used to obtain satisfactory postoperative analgesia in the first few hours after surgery in all three groups, which is consistent with previous studies.

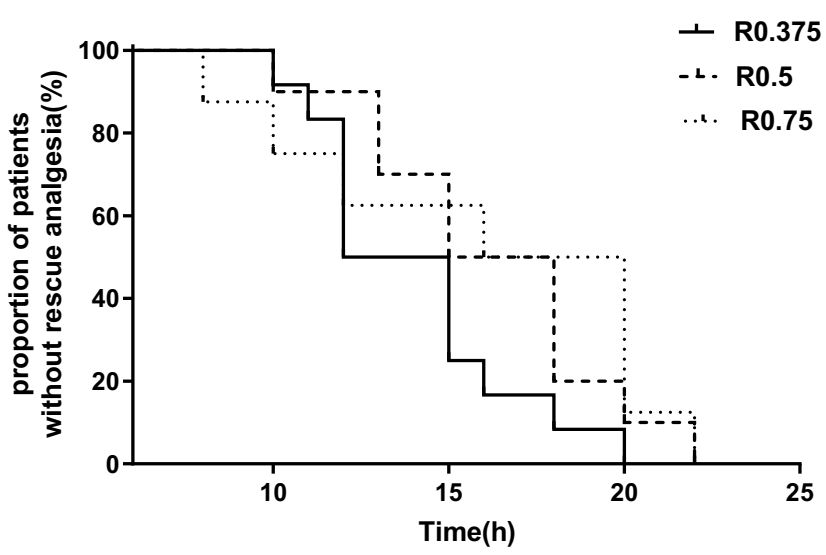

Figure 5 The time of first need for tramadol. Kaplan-Meier survival curves demonstrated that the time between completion of surgery and the first administration of rescue analgesia. R0.375: $0.375 \%$ ropivacaine with $20 \mathrm{~mL}$ volume; R0.5: $0.5 \%$ ropivacaine with $20 \mathrm{~mL}$ volume; $\mathrm{R} 0.75: 0.75 \%$ ropivacaine with $20 \mathrm{~mL}$ volume. There was no significant difference among the three groups, $P=0.180$.

The major determinants of the pharmacodynamics of LAs remain controversial. One study reported that reducing LAs concentration or dose could shorten blockade duration. ${ }^{10}$ We used cold stimulus to evaluate the duration of blockade after SAP block with different ropivacaine concentrations, and the results were consistent with previous reports. Although a small amount of LAs may reduce LAs-related complications, continuous catheterization may be required to prolong blockade duration with a low concentration of LAs in order to meet postoperative analgesia needs. ${ }^{16}$ Compared with a single-shot block, continuous LAs infusion may increase the risk of catheterrelated infections ${ }^{17,18}$ and delay the patient's early postoperative activity. Therefore, we expected to determine the appropriate LAs concentration for a single-shot block to 
satisfy postoperative analgesia requirements without increasing the additional risk of anesthesia.

The postoperative effect of SAP block with $0.375 \%$ ropivacaine was inferior to that of $0.5 \%$ or $0.75 \%$ ropivacaine, which may be related to a shorter blockade duration. We observed that although $0.75 \%$ ropivacaine had a longer duration than $0.5 \%$ ropivacaine, it did not lead to a better analgesia effect. This may be due to the fact that the mean durations of $0.5 \%$ and $0.75 \%$ concentrations are $>10 \mathrm{~h}$, which already cover the worst time of postoperative pain after breast surgery. Subtle differences in pain scores are not easily detected. A study about the relationship between LAs concentration in regional anesthesia and enhanced recovery after surgery confirmed that $0.5 \%$ ropivacaine was an optimal concentration that could reduce LAs toxicity without sacrificing analgesia. ${ }^{19}$ Therefore, we propose that $0.5 \%$ ropivacaine is a suitable LAs concentration for SAP block.

We studied blockade efficacy in patients undergoing benign breast tumor (eg, fibroadenoma or intraductal papilloma) resection based on the following factors. Firstly, previous studies on SAP block mostly focused on thoracoscopic surgery or mastectomy for breast cancer, the surgical incision location affects dermatomal paresthesia, whereas the incision required for benign breast tumor resection in the breast or areola is limited and does not affect the evaluation of blockade duration. Secondly, outpatient surgery continues to expand in scope and volume, and breast surgery may be performed as an outpatient procedure, which requires simple and effective analgesia management to support rapid recovery and shorten hospital stay. Regional analgesia alone or in combination with general anesthesia have been recommended for outpatient surgery. ${ }^{20,21}$ SAP block is a type of regional anesthesia, especially the single-shot SAP block, which is more suitable for benign breast tumor resection. Although we observed a significant difference in the duration of sensory block among the three groups and the NRS scores in Group $0.375 \%$ were significantly higher than that in the Groups $0.5 \%$ and $0.75 \%$, there was no difference in the time of first request for rescue analgesics or total rescue analgesic consumption. This could be attributed to the small incision required for benign breast tumor resection and limited postoperative pain.

Cold or pain stimuli are commonly used to detect sensory block. Børglum et al reported that both testing methods yielded similar results when assessing the efficacy of transverse abdominis plane blocks. ${ }^{22}$ Previous studies used cold stimuli to assess sensory block duration after regional anesthesia with the aim of reducing patient discomfort, ${ }^{12,23}$ so we employed the same approach.

This study had several limitations: 1) There was no concentration gradient, which was to reduce the number of groups and false-negative results from multiple comparisons. 2) We did not include a control group based on considering the needs of analgesia and the maximum patient benefit. 3) Clinical use of adjuvants may prolong the blocking effect of lowconcentration ropivacaine, but that was not assessed in this study. 4) Plasma ropivacaine levels at different concentrations were not measured. Although previous studies have not reported any adverse reactions associated with SAP block, higher concentrations may carry a greater risk. ${ }^{24}$ The results should be confirmed in a study with longer and more invasive follow-up and a larger study population.

\section{Conclusions}

A comparison of $0.5 \%$ and $0.75 \%$ ropivacaine showed no significant difference in postoperative analgesia, but both were superior to $0.375 \%$ ropivacaine, although higher ropivacaine concentration lengthened the duration of SAP block. Therefore, SAP block with $0.5 \%$ ropivacaine is recommended for postoperative analgesia in breast surgery.

\section{Abbreviations}

ASA, American Society of Anesthesiologists; AUC, area under the curve; BMI, body mass index; LAs, local anesthetics; NRS, numerical rating scale; SAP, serratus anterior plane.

\section{Ethical Statement}

The authors declare that all patients gave written informed consent before initiation of the study protocol and was conducted in accordance with the Declaration of Helsinki. The study was approved by the Ethics Committee of The First Affiliated Hospital of Wenzhou Medical University (No. 12, 2018).

\section{Data Sharing Statement}

One of the corresponding authors (wzshikejian@163.com) will be ready to send all related data for any authority on request by an email message. The data will be available this way for up to 6 months after the paper is published.

\section{Funding}

This work is supported by the National Natural Science Foundation of China (Grant No. 81900231, Beijing, 
China), and by Wenzhou Science and Technology Bureau (Y20190519, Wenzhou, China).

\section{Disclosure}

The authors report no conflicts of interest in this work.

\section{References}

1. Biswas A, Castanov V, Li Z, et al. Serratus plane block: a cadaveric study to evaluate optimal injectate spread. Reg Anesth Pain Med. 2018;43(8):854-858. doi:10.1097/AAP.0000000000000848

2. Mazzinari G, Rovira L, Casasempere A, et al. Interfascial block at the serratus muscle plane versus conventional analgesia in breast surgery: a randomized controlled trial. Reg Anesth Pain Med. 2019;44 (1):52-58. doi:10.1136/rapm-2018-000004

3. Semyonov M, Fedorina E, Grinshpun J, et al. Ultrasound-guided serratus anterior plane block for analgesia after thoracic surgery. $J$ Pain Res. 2019;12:953-960. doi:10.2147/JPR.S191263

4. Wang L, Wang Y, Zhang X, Zhu X, Wang G. Serratus anterior plane block or thoracic paravertebral block for postoperative pain treatment after uniportal video-assisted thoracoscopic surgery: a retrospective propensity-matched study. J Pain Res. 2019;12:2231-2238. doi:10.2147/ JPR.S209012

5. Barbera C, Milito P, Punturieri M, Asti E, Bonavina L. Serratus anterior plane block for hybrid transthoracic esophagectomy: a pilot study. J Pain Res. 2017;10:73-77. doi:10.2147/JPR.S121441

6. Biswas A, Luginbuehl I, Szabo E, Caldeira-Kulbakas M, Crawford MW, Everett T. Use of serratus plane block for repair of coarctation of aorta: a report of 3 cases. Reg Anesth Pain Med. 2018;43(6):641-643. doi:10.1097/AAP.0000000000000801

7. Kunigo T, Murouchi T, Yamamoto S, Yamakage M. Injection volume and anesthetic effect in serratus plane block. Reg Anesth Pain Med. 2017;42(6):737-740. doi:10.1097/AAP.0000000000000649

8. Blanco R, Parras T, McDonnell JG, Prats-Galino A. Serratus plane block: a novel ultrasound-guided thoracic wall nerve block. Anaesthesia. 2013;68(11):1107-1113. doi:10.1111/anae.2013.68.issue-11

9. Ilfeld BM, Moeller LK, Mariano ER, et al. Continuous peripheral nerve blocks: is local anesthetic dose the only factor, or do concentration and volume influence infusion effects as well? Anesthesiology. 2010;112(2):347-354. doi:10.1097/ALN.0b013e3181ca4e5d

10. Fenten MG, Schoenmakers KP, Heesterbeek PJ, Scheffer GJ, Stienstra R. Effect of local anesthetic concentration, dose and volume on the duration of single-injection ultrasound-guided axillary brachial plexus block with mepivacaine: a randomized controlled trial. $B M C$ Anesthesiol. 2015;15:130. doi:10.1186/s12871-015-0110-0

11. Wong AK, Keeney LG, Chen L, Williams R, Liu J, Elkassabany NM. Effect of local anesthetic concentration $(0.2 \%$ vs $0.1 \%$ ropivacaine) on pulmonary function, and analgesia after ultrasound-guided interscalene brachial plexus block: a randomized controlled study. Pain Med. 2016;17(12):2397-2403. doi:10.1093/pm/pnw057
12. Chen Y, Shi K, Xia Y, et al. Sensory assessment and regression rate of bilateral oblique subcostal transversus abdominis plane block in volunteers. Reg Anesth Pain Med. 2018;43(2):174-179. doi:10.1097/ AAP.0000000000000715

13. Davies F, Gladstone RJ, Stibbe EP. The anatomy of the intercostal nerves. J Anat. 1932;66(Pt 3):323-333.

14. Khemka R, Chakraborty A. Ultrasound-guided modified serratus anterior plane block for perioperative analgesia in breast oncoplastic surgery: a case series. Indian J Anaesth. 2019;63(3):231-234. doi:10.4103/ija.IJA_752_18

15. Abdallah FW, MacLean D, Madjdpour C, Cil T, Bhatia A, Brull R. Pectoralis and serratus fascial plane blocks each provide early analgesic benefits following ambulatory breast cancer surgery: a retrospective propensity-matched cohort study. Anesth Analg. 2017;125(1):294-302. doi:10.1213/ANE.0000000000001975

16. O’Scanaill P, Keane S, Wall V, Flood G, Buggy DJ. Single-shot pectoral plane (PECs I and PECs II) blocks versus continuous local anaesthetic infusion analgesia or both after non-ambulatory breast-cancer surgery: a prospective, randomised, double-blind trial. Br J Anaesth. 2018;120(4):846-853. doi:10.1016/j.bja.2017.11.112

17. Gabriel RA, Ilfeld BM. Percutaneous peripheral nerve stimulation and other alternatives for perineural catheters for postoperative analgesia. Best Pract Res Clin Anaesthesiol. 2019;33(1):37-46. doi:10.1016/j.bpa.2019.02.002

18. Bomberg H, Kubulus C, Herberger S, et al. Tunnelling of thoracic epidural catheters is associated with fewer catheter-related infections: a retrospective registry analysis. Br J Anaesth. 2016;116(4):546-553. doi:10.1093/bja/aew026

19. Ping C, Lin QS, Lin XZ. Optimal concentration of the transversus abdominis plane block in enhanced recovery after surgery protocols for patients of advanced age undergoing laparoscopic rectal cancer surgery. J Int Med Res. 2018;46(11):4437-4446. doi:10.1177/0300060518790699

20. Liu SS, Strodtbeck WM, Richman JM, Wu CL. A comparison of regional versus general anesthesia for ambulatory anesthesia: a meta-analysis of randomized controlled trials. Anesth Analg. 2005;101(6):1634-1642. doi:10.1213/01.ANE.0000180829.70036.4F

21. Boezaart AP, Davis G, Le-Wendling L. Recovery after orthopedic surgery: techniques to increase duration of pain control. Curr Opin Anaesthesiol. 2012;25(6):665-672. doi:10.1097/ACO.0b013e328359ab5a

22. Borglum J, Jensen K, Christensen AF, et al. Distribution patterns, dermatomal anesthesia, and ropivacaine serum concentrations after bilateral dual transversus abdominis plane block. Reg Anesth Pain Med. 2012;37(3):294-301. doi:10.1097/AAP.0b013e31824c20a9

23. Stoving K, Rothe C, Rosenstock CV, Aasvang EK, Lundstrom LH, Lange KH. Cutaneous sensory block area, muscle-relaxing effect, and block duration of the transversus abdominis plane block: a randomized, blinded, and placebo-controlled study in healthy volunteers. Reg Anesth Pain Med. 2015;40(4):355-362. doi:10.1097/AAP.0000000000000252

24. Murouchi T, Iwasaki S, Yamakage M. Chronological changes in ropivacaine concentration and analgesic effects between transversus abdominis plane block and rectus sheath block. Reg Anesth Pain Med. 2015;40(5):568-571. doi:10.1097/AAP.0000000000000288
Journal of Pain Research

\section{Publish your work in this journal}

The Journal of Pain Research is an international, peer reviewed, open access, online journal that welcomes laboratory and clinical findings in the fields of pain research and the prevention and management of pain. Original research, reviews, symposium reports, hypothesis formation and commentaries are all considered for publication. The manuscript management system is completely online and includes a very quick and fair peer-review system, which is all easy to use. Visit http:// www.dovepress.com/testimonials.php to read real quotes from published authors. 